CAPÍTULO 5

\title{
NUTRACÉUTICOS Y ALIMENTOS FUNCIONALES: UNA REVISIÓN DE OPORTUNIDADES
}

\author{
Claudia Estela Restrepo Flórez'1, Hilda Helena Estrada-López², \\ Hernán Guillermo Saumett-España ${ }^{3}$
}

1 Investigadora Junior, Magíster en Ciencias Farmacéuticas y Alimentarias, vinculada al Instituto de Ciencia y Tecnología Alimentaria - INTAL Itagüí (Colombia). crestrepo@fundacionintal.org

2 Administradora de Empresas, Universidad Autónoma del Caribe. Doctora en Administración de Empresas, Universidad Autónoma de Querétaro, México. Investigadora del Grupo Innovación y Desarrolo Empresarial-GIDE. Universidad Simón Bolívar, Barranquilla (Colombia). Docente en la Facultad de Ciencias Económicas de la Universidad del Atlántico, Barranquilla (Colombia). hileslo@hotmail.co

3 Sociólogo, Universidad Simón Bolívar. Magíster en Proyectos de desarrollo social, Universidad del Norte. Docente-Investigador del Grupo Innovación y Desarrollo Empresarial-GIDE. Úniversidad Simón Bolívar, Barranquilla (Colombia).

herse47t@unisimonbolivar.edu.co 


\section{RESUMEN}

A nivel mundial, más personas están preocupadas por la salud e interesadas en las denominadas enfermedades prevenibles, como la cardíaca, cáncer, osteoporosis, entre otras. Junto con la expansión de las fuentes de información, surgen más y más artículos en revistas dedicados a la relación que hay entre la dieta y la salud, y específicamente, al concepto nutracéutico. La industria de alimentos está aprovechando esto, reconociendo un mercado potencial e invirtiendo en investigación de compuestos nutracéuticos, desarrollo de nuevos productos y mercadeo. Estos productos caen en una categoría más grande llamada alimentos funcionales, que son naturales o manufacturados que contienen compuestos bioactivos que pueden influenciar positivamente la salud humana. Los alimentos funcionales no solo incluyen alimentos nutracéuticos, sino que también incluyen alimentos diseñados especialmente para mejorar el desempeño, por ejemplo, de tipo cognitivo o físico.

Existe otro concepto emergente relacionado con los nutracéuticos, en los cuales se cree que los alimentos completos probablemente son más poderosos que los componentes individuales o los extractos puros de sustancias. Este capítulo plantea una revisión a los conceptos nutracéutico, alimentos funcionales y el potencial de seis productos de la tradición agrícola del sur del Atlántico colombiano.

Palabras clave: Alimentos funcionales, compuestos bioactivos, nutracéuticos, productos agrícolas.

\section{ABSTRACT}

At present, worldwide more and more people is concerned about health and they care about preventable diseases such as cardiovas- 
cular disease, cancer, osteoporosis, and so on. With the information sources expansion, there are more and more articles in journals devoted to explain the relation between diet and health, and specifically on the nutraceutical concept. Food industry is taking advantage of this, recognizing a potential market and investing on research of nutraceutical compounds, development and marketing of new products. These products fall on a greater category called functional foods, this are naturals or manufactured foods that contain bioactive compounds that can positively influence human health. Functional foods include not only nutraceutical food, but specially designed food to enhance performance, such as, cognitive or physical.

There is another emerging concept related to nutraceuticals, where it's believed that the complete food is more effective than individual compounds or the pure substance extract. This chapter presents a review for nutraceutical concept, functional food and the potential of six traditional agricultural food from the Colombian south Atlántico.

Key words: Functional foods, bioactive compounds, nutraceuticals, agricultural products.

\section{INTRODUCCIÓN}

Los patrones de la alimentación tradicional se están modificando hacia el consumo de alimentos no tradicionales en las diferentes regiones del mundo. La preocupación global por la relación entre la dieta y la salud, ha centrado la atención en la obesidad como enfermedad metabólica caracterizada por un aumento del tejido adiposo por encima de ciertos límites, que se manifiesta por alteraciones morfológicas y sobrepeso (Malik, Willett y Hu, 2013). 
Los problemas de salud pública relacionados con los malos hábitos alimenticios están relacionados también con otros factores socioeconómicos culturales. Una consecuencia del cambio de hábitos es la reducción de tiempo dedicada a la preparación de alimentos, para lo cual la industria de los alimentos intenta suplir esta falta, procesando total o parcialmente el alimento, y se ofrece al consumidor de una forma que sea conveniente para él (Pérez-Álvarez, Fernández-López, y Sayas-Barberá, 2003).

Las sociedades occidentales se ven avocadas el envejecimiento de la población como consecuencia de los avances en la medicina, la generalización de hábitos de vida sanos, la nutrición, la higiene y ejercicio físico. Estos factores han incrementado la esperanza de vida, lo que ha aumentado, a su vez, el interés en la identificación de factores que conduzcan a un envejecimiento saludable (Godfray et al., 2010; Pérez-Álvarez, et al., 2003).

Nuevos factores socioculturales como el aumento del poder de compra, la proliferación de establecimientos de "comida rápida" o el incremento de mujeres trabajadoras fuera del hogar, entre otros, han hecho posible la instalación de prácticas que caracterizan el comportamiento alimentario de los jóvenes. Algunas de estas prácticas son: la omisión de algunas de las principales ingestas alimentarias, en particular la del desayuno; la frecuencia en el consumo de snacks, o la elevada ingesta de bebidas refrescantes (Pérez-Álvarez, et al., 2003).

En la última etapa del siglo XX y en los inicios del XXI, se están introduciendo nuevos estilos de vida que, en parte, son el fruto 
del avance científico, la biotecnología, tecnologías de información, innovaciones industriales y comerciales, combinado con una apertura de las fronteras y una difusión a gran velocidad de la información. Todos estos factores inciden en la alimentación, y han contribuido a la emergencia de una nueva categoría de productos que no pueden clasificarse como alimentos tradicionales, y que se han denominado "nuevos alimentos".

En la jerga de la producción de alimentos, ha surgido una comprometedora industria que se ha organizado con el objetivo de responder los deseos de los consumidores. Ante esta situación se comienzan a discriminar los alimentos en función de su finalidad de la siguiente manera:

1. Relacionados con salud: alimentos funcionales, nutracéuticos, suplementos vitamínicos, dietarios y ecológicos.

2. Relacionados al estilo de vida: los alimentos básicos, productos estándar de base, fast food, comida preparada.

3. Relacionados a la cultura: los alimentos auténticos y autóctonos.

Los conceptos básicos en nutrición están experimentando un cambio significativo. Se supera la idea tradicional de una "dieta adecuada" en el solo sentido de aportar nutrientes suficientes que aseguren la supervivencia de un individuo, satisfacer sus necesidades metabólicas y complacer placenteramente su sensación de hambre y bienestar. Hoy, además de ello y de lo relativo a la seguridad alimentaria, el énfasis se acentúa en la potencialidad de los alimentos para la mejora de la salud, del bienestar, y reducir el riesgo de enfermedades. Así el concepto de "nutrición adecuada" tiende a ser sustituido por "nutrición óptima", en cuyo ámbito aparecen los "alimentos funcionales" (Pérez-Álvarez, et al., 2003). 
Esta nueva nutrición médica, es decir, la nueva orientación para la prevención de enfermedades, abre una caja de oportunidades y riesgos. La posibilidad de orientar alimentos al tratamiento de problemas de salud es toda una realidad, pero han surgido a la vez cuestionamientos sobre la seguridad en sus formas concentradas (extractos), relación dosis-efecto, la autenticidad, y aspectos éticos.

Un gran interés se ha dado al sector de los alimentos para satisfacer la búsqueda del consumidor de una vida larga y saludable, libre de enfermedades debilitantes, al desarrollar productos enriquecidos o fortificados con nutracéuticos. La comunidad científica y fabricantes de alimentos están interesados en identificar estos ingredientes no nutritivos que combaten enfermedades, y en desarrollar productos que los contengan, debido al potencial económico que representan (Farmer, 2005; Prabu, Suriyaprakash, Kumar, Sureshkumar, y Ragavendran, 2012).

Los fabricantes quieren informar tanto a los clientes como a los consumidores de los beneficios de los productos fortificados. Para ello, las regulaciones de etiquetado y publicidad, deben proteger al consumidor de la confusión y tergiversación de los beneficios para la salud que las etiquetas y publicidad de las compañías declaran. Con la amplia disponibilidad de nutracéuticos, fitoquímicos, o productos mejorados con fuentes herbales, surge la advertencia de profesionales de la salud de los peligros que algunos nutracéuticos representan para la población con medicamentos prescritos, y población infantil consumidora de golosinas y snacks enriquecidos (Farmer, 2005; Prabu et al., 2012; Shahidi, 2009).

La oferta comercial se da de varias formas, y las compañías de in- 
gredientes obtienen extractos concentrados de fitoquímicos que se adaptan a estos sistemas alimentarios. En el mercado se observan golosinas pretendiendo prevenir el cáncer, reforzar el sistema inmune, reducir los niveles de colesterol; aportes importantes de fibra y calcio en jugos de frutas; preparaciones de té fortificadas con polifenoles; chips de papas, maíz y otros añadidos de extractos herbales y de plantas, como por ejemplo: el ginseng (promotor de longevidad), ginkgo biloba (mejorador de la memoria), entre otros. Cada declaración es precedida de la expresión "se atribuye" (Farmer, 2005; Prabu et al., 2012; Shahidi, 2009).

Los fitoquímicos son materiales biológicamente activos. Así como las compañías farmacéuticas se han propuesto usarlos en la preparación de medicinas, o el uso de la molécula base para preparar medicinas más eficazmente, están siendo extraídos de sus fuentes naturales y concentrados por proveedores, y vendidos como extractos al público general o a los fabricantes de productos de consumo para la adición a alimentos procesados. Su seguridad en alimentos preparados a concentraciones mayores que las naturalmente encontradas en una fuente alimentaria, no han podido ser establecidas para todos los segmentos de la población. De hecho, falta evidencia para demostrar si lo más conveniente es fortificar los alimentos con el componente activo o con el extracto completo. Su presencia en diversidad de productos, en los cuales normalmente no se encuentran, pero han sido añadidos, puede ser igualmente perjudicial para alguna población que no consume suficientes alimentos de este tipo. Por ejemplo, snacks enfocados a niños o jóvenes, quienes son consumidores voraces, pudieran no resultar vehículos muy apropiados para la suplementación con algunos fitoquímicos (Farmer, 2005; Prabu et al., 2012; Shahidi, 2009). 


\section{CONCEPTO E HISTORIA DE LOS NUTRACÉUTICOS}

El campo de los nutracéuticos, como un campo médico/nutricional, está captando la atención de los profesionales de la nutrición y del cuidado de la salud. Muchos aspectos de los nutracéuticos parecían estar bajo la sombra de la "medicina alternativa". Hace un par de décadas, los jóvenes científicos fueron desalentados para continuar investigando en temas como los flavonoides, ya que era criticada su importancia práctica. Aún en estos tiempos, muchas de estas áreas aparecen bajo estudio de la medicina alternativa, tales como extractos herbales, y se están incluyendo en el rango de terapias médicas convencionales. Los grupos de investigación están dedicando sus programas a tópicos nutracéuticos, mientras que otros investigadores que se enfocaban en tópicos nutricionales más tradicionales, están ampliando sus parámetros de investigación para incluir investigaciones nutracéuticas. Para continuar con el capítulo debemos definir nutracéutico y Alimento Funcional Wildman, 2001; Bagchi, Preus y Swaroop, 2016; Kaur y Das, 2011.

Nutracéutico: Compuestos químicos encontrados como un componente natural de los alimentos $u$ otro producto comestible, que han sido hallados como benéficos para el cuerpo humano, previniendo, tratando una o más enfermedades, o mejorando el desempeño fisiológico. Nutrientes esenciales pueden ser considerados nutracéuticos si proveen beneficios más allá de su papel esencial en el crecimiento normal o mantenimiento del cuerpo humano. Un ejemplo son las propiedades antioxidantes de las vitaminas $\mathrm{C}$ y $\mathrm{E}$ (Prabu et al., 2012; Shahidi, 2009).

Alimento Funcional: Un alimento, natural o formulado, que mejorará el desempeño fisiológico, o previene/trata enfermedades 
o desórdenes. Los alimentos funcionales incluyen aquellos ítems desarrollados con propósitos tanto para la salud como para el desempeño físico. La mesa del Instituto de Alimentos Medicinales y Nutrición definió como alimentos funcionales a "cualquier alimento o ingrediente alimentario que puede proveer un beneficio a la salud más allá de los nutrientes tradicionales que contiene"(Kaur y Das, 2011, p.861).

Los nutracéuticos son componentes de los alimentos exóticos y tradicionales que tienen el potencial de mejorar la salud humana. Estas sustancias pueden ser: (1) parte de un alimento fresco completo; (2) parte de un alimento procesado; (3) una sustancia fortificada o enriquecida en un alimento, o (4) proveerse como suplemento. Los nutracéuticos son compuestos que se encuentran en las plantas, animales, levaduras, hongos y bacterias. Esto no quiere decir que los alimentos funcionales futuros no incluirán variantes sintéticas de los nutracéuticos naturales. Si la sustancia está relacionada con una planta a menudo se conoce como fitoquímico. Algunas veces el término botánica medicinal se usa como sinónimo de fitoquímico (Wildman, 2001).

\section{CLASES DE NUTRACÉUTICOS}

A continuación se presenta un resumen de algunas clases de nutracéuticos. Desde el punto de vista teleológico, las plantas y otras formas de vida existen en el planeta con los mismos objetivos básicos que los humanos, entre los cuales se tiene la defensa. (Bagchi et al., 2016; Keservani et al., 2010; Prabu et al., 2012; Wildman, 2001). 


\section{Carotinoides}

Los carotinoides son una amplia categoría de moléculas provenientes de plantas, que incluyen los carotenos y xantofilas; hacen parte de una clase mayor de moléculas llamadas terpenoides o isoprenoides. Los carotinoides, como pigmento, tienen la capacidad de absorber la luz visible y aparecen coloreados, mientras que su papel nutracéutico en los humanos se relaciona principalmente con protección molecular contra el ataque de radicales libres (Wildman, 2001).

\section{Ácido Linoleico Conjugado}

El ácido linoleico conjugado (CLA) se encuentra básicamente en la carne y leche. Cierta evidencia experimental sugiere que el CLA tiene propiedades anticarcinogénicas, puede disminuir el avance de ateroesclerosis y puede estimular eventos del sistema inmune. Otra evidencia sugiere que el CLA puede inhibir la lipogénesis, mientras que también puede incrementar algunos mecanismos involucrados en el uso de ácidos grasos in vivo. Es producido por una bacteria específica en el rumen de los animales por modificación del ácido linoleico de su dieta, y luego es absorbida por los rumiantes y entra a los tejidos, incluyendo el tejido mamario y el músculo esquelético (Wildman, 2001).

\section{Flavonoides}

Los flavonoides son una amplia categoría de compuestos con capacidad antioxidante, producidos por plantas, muchos parecen tener efectos nutracéuticos potenciales al disminuir los niveles de colesterol, eventos de osteoporosis y cancerígenos (Bagchi et al., 2016). 


\section{Derivados de Aminoácidos que contienen Nitrógeno y Azufre}

Las plantas producen metabolitos secundarios que contienen nitrógeno. Entre estas estructuras se encuentran los alcaloides y glucósidos cianogénicos, que son derivados de aminoácidos comunes. Los alcaloides aparecen en el 20 \% de las especies de plantas vasculares. Estas sustancias, como las altamente reconocidas cocaína, nicotina, morfina y cafeína, se caracterizan por su notable efecto fisiológico en los vertebrados. En algún momento se pensó que los alcaloides eran un sistema de almacenamiento y desecho de nitrógeno. Sin embargo, ahora los científicos creen que los alcaloides son moléculas para defenderse de los depredadores, especialmente los mamíferos, debido a su toxicidad (Bagchi et al., 2016; Wildman, 2001).

Los capsaicinoides son estructuras alcaloides producidas por frutas de pimientos, y son derivados de los aminoácidos fenilalanina y valina o leucina, y de cadenas ramificadas de ácidos grasos. La capsaicina se usa medicinalmente para tratar muchas condiciones médicas, incluyendo artritis, y pueden tener propiedades anticarcinogénicas y antioxidantes. Los capsaicinoides pueden irritar la superficie cutánea y producir sensación "picante" cuando se consume, por interacción con receptores del dolor, lo que puede disuadir a los animales de su consumo. Mientras que algunos vegetales crucíferos contienen glucosinolatos que en sí mismos son inertes, pero pueden convertirse en metabolitos tóxicos cuando los tejidos de la planta sufren traumas y los contenidos de diferentes tejidos se mezclan. Entre las sustancias derivadas que aparecen se tienen isotiocianatos, tiocianatos y nitrilos que se producen cuando el tejido de la planta sufre traumatismos y permite que los glucosinolatos de ciertas células se mezclen con enzimas en otras células (Wildman, 2001). 


\section{Inhibidores de Proteinasa y $\alpha$-amilasa.}

Entre el arsenal defensivo de algunas plantas están los inhibidores de proteasas o proteinasas. Los inhibidores de BowmanBirk (BBI) son unas sustancias presentes en la soya, y se cree que tienen propiedades anticarcinogénicas. Los inhibidores de proteasas son mecanismos de defensa de las plantas. Por ejemplo, los tomates producirán estas sustancias para dificultar la actividad enzimática de la proteína digestiva de los herbívoros e insectos. Además de los inhibidores de proteasas, los tejidos vegetales también producen inhibidores de $\alpha$-amilasa que inhiben la actividad de la $\alpha$-amilasa digestiva sobre el almidón. Las plantas también producen sustancias llamadas lectinas, sustancias que se enlazan a los carbohidratos y proteínas que contienen carbohidratos, e interfieren con la absorción de nutrientes en el intestino de los animales (Wildman, 2001).

\section{Ácidos Grasos Poliinsaturados omega 3 (omega-3 PUFA)}

La clasificación nutracéutica de los w-3 PUFA está basada en su relación inversa con la enfermedad coronaria, y ciertos tipos de cáncer y desórdenes inflamatorios como la artritis. Los humanos consumen w-3 PUFA principalmente de plantas y aceites de pescado, lo que sugiere que tanto plantas como animales pueden producir estos PUFAS. Sin embargo, los animales no poseen las enzimas para producir las insaturaciones necesarias para producir las w-3 PUFA; por lo tanto, los w-3 PUFA encontrados en los pescados y otros animales marinos provienen de su dieta. Estas moléculas son lípidos polares que sirven como los lípidos estructurales en las membranas vegetales (Wildman, 2001). Hay por lo menos seis diferentes ácidos grasos empleados por las plantas, estas incluyen: ácido palmítico (16.0), ácido linoleico (18:2 w-6) y ácido linolénico (18:3 w-3). 


\section{Terpenoides}

Muchos de los terpenoides y sus derivados son agentes tóxicos para los insectos. De hecho, algunos de estos monoterpenos, tales como los esteres de monoterpeno llamados piretroides, se usan como componentes de insecticidas comerciales. La mayoría de las plantas contienen los llamados aceites esenciales, que son una mezcla de monoterpenos y sesquiterpenos volátiles; tienen propiedades repelentes de insectos y se encuentran en las vellosidades glandulares de la epidermis o en la piel de las frutas. El compuesto nutracéutico altamente promocionado limoneno se encuentra en los aceites esenciales de la piel de los cítricos. El mentol es el principal monoterpeno en el aceite esencial de la menta. Como un caso particular, muchas plantas liberan ciertos monoterpenos y sesquiterpenos después de que el insecto ha comenzado a alimentarse, estos terpenoides no son tóxicos para el insecto, pero sirven para atraer los predadores de estos insectos. Adicionalmente, una gran cantidad de diterpenos han mostrado ser toxinas e irritantes para la piel que disuaden herbívoros e insectos (Wildman, 2001).

\section{CLASIFICACIÓN DE LOS NUTRACÉUTICOS}

El enfoque de los nutracéuticos dependerá de los intereses de las diferentes ramas de la ciencia, ya que un cardiólogo estará más interesado en el efecto positivo sobre la hipertensión y la hipocolesterolemia; los oncólogos estarán más interesados en las sustancias con una actividad anticarcinogénica, y los científicos de alimentos que trabajan en alimentos funcionales no solo se interesan en las propiedades fisiológicas, sino en las propiedades sensoriales y de estabilidad, costo y eficiencia. Por ejemplo, el anticarcinogénico limonina triterpeno es liposoluble e intensamente amargo, lo cual limita su uso como ingrediente de un alimento funcional, mien- 
tras que el glucósido derivado de la limonina comparte algo de su actividad anticarcinogénica, es soluble en agua y prácticamente sin sabor, potencializando su uso como ingrediente (Bagchi et al., 2016; Keservani et al., 2010; Wildman, 2001).

Los nutracéuticos se pueden organizar de muchas diferentes formas, dependiendo del interés o necesidad específica. A continuación, se describen varias formas de clasificar los nutracéuticos dependiendo de: fuente alimentaria, mecanismo de acción y naturaleza química (Wildman, 2001, 2007).

\section{CLASIFICACIÓN POR FUENTE ALIMENTARIA}

\section{Fuentes animales, plantas y microbianas}

Uno de los modelos más usados para clasificar los nutracéuticos es su fuente alimentaria, y pueden dividirse en los siguientes grupos: plantas, animales o microbianas (bacterias y levaduras). Esta clasificación es útil desde el punto de vista educativo, pero como ya se planteó el caso del CLA que es parte de la dieta humana, y se encuentra principalmente en carnes y productos lácteos, pero en realidad es producido por bacterias en el rumen de la vaca. Muchos de los compuestos nutracéuticos se encuentran en animales, plantas y microorganismos, pero en algunos casos, como el de la colina, las plantas y animales son su mejor fuente (Wildman, 2007). 
NUTRACÉUTICOS Y ALIMENTOS FUNCIONALES:

una revisión de oportunidades

Tabla 20

Ejemplos de sustancias nutracéuticas agrupadas por fuentes alimentarias

\begin{tabular}{|c|c|c|}
\hline Plantas & Animal & Microbiana \\
\hline$\beta$-glucano & Ácido linoleico conjugado (CLA) & $\begin{array}{l}\text { Saccharomyces boulardii } \\
\text { (levadura). }\end{array}$ \\
\hline Ácido ascórbico & Ácido Eicosapentaenoico (EPA) & Bifidobacterium bifidum. \\
\hline g-tocotrienol & Ácido Docosahexaenoco (DHA) & B. longum; B. infantis. \\
\hline Quercetina & Esfingolípidos & $\begin{array}{l}\text { Lactobacillus acidophilus } \\
\text { (LC1). }\end{array}$ \\
\hline Luteolina & Colina & L. acidophilus (NCFB 1748). \\
\hline Celulosa & Lecitina & $\begin{array}{l}\text { Streptococcus salvarius (subs. } \\
\text { Thermophilus). }\end{array}$ \\
\hline Luteína & Calcio & \\
\hline Ácido gálico & Coenzima Q10 & \\
\hline $\begin{array}{l}\text { Alcohol perillí- } \\
\text { lico }\end{array}$ & Selenio & \\
\hline Indol-3-carbonol & Zinc & \\
\hline Pectina & Creatina & \\
\hline Daidzeína & Minerales & \\
\hline \multicolumn{3}{|l|}{ Glutatión } \\
\hline \multicolumn{3}{|l|}{ Potasio } \\
\hline \multicolumn{3}{|l|}{ Alicina } \\
\hline \multicolumn{3}{|l|}{ d-limoneno } \\
\hline \multicolumn{3}{|l|}{ Genisteína } \\
\hline \multicolumn{3}{|l|}{ Licopeno } \\
\hline \multicolumn{3}{|l|}{ hemicelulosa } \\
\hline \multicolumn{3}{|l|}{ Lignina } \\
\hline \multicolumn{3}{|l|}{ Capsaicina } \\
\hline \multicolumn{3}{|l|}{ Geraniol } \\
\hline \multicolumn{3}{|l|}{$\beta$-ionona } \\
\hline \multicolumn{3}{|l|}{$\beta$-tocoferol } \\
\hline \multicolumn{3}{|l|}{$\beta$-caroteno } \\
\hline \multicolumn{3}{|l|}{ Selenio } \\
\hline \multicolumn{3}{|l|}{ Zeaxantina } \\
\hline Minerales & & \\
\hline
\end{tabular}




\section{Clasificación por mecanismo de acción}

Otra forma de clasificar los nutracéuticos es por su mecanismo de acción. Este sistema agrupa los nutracéuticos sin importar su fuente alimentaria, basado en sus propiedades fisiológicas probadas o atribuidas. Entre estas estarían: antioxidantes, antibacterianas, hipotensivas, hipocolesterolémicas, antiinflamatorias, anticarcinogénicas, osteoprotectoras, entre otras. Algunos ejemplos de estas se dan en la Tabla 21. Sin embargo, muchos temas relacionados con la toxicidad, sinergismo y competición, asociados con los nutracéuticos y los alimentos, todavía no se conocen bien (Wildman, 2001, 2007).

Lo que puede ser interesante es que hay varios nutracéuticos que pueden listarse y presentar más de un mecanismo de acción. Una de las familias más versátiles es la de los w-3 PUFA; sus propiedades nutracéuticas pueden estar relacionadas con sus efectos directos e indirectos. Por ejemplo, estos ácidos grasos son usados como precursores de sustancias eicosanoides que actúan localmente produciendo vasodilatación, broncodilatación, y evitan la aglutinación de plaquetas y la formación de coágulos. Estas funciones pueden ser profilácticas para el asma y enfermedades coronarias. Los Omega-3 PUFA también pueden reducir la actividad de la proteína quinasa C y la tirosina quinasa, ambas involucradas en el mecanismo de señalización del crecimiento celular. El efecto directo de estos ácidos grasos puede reducir la hipertrofia cardíaca y la proliferación de células cancerígenas. Los Omega-3 PUFA también parecen inhibir la síntesis de la ácido-graso-sintetasa (FAS), el complejo enzimático principal involucrado en la síntesis de nuevos ácidos grasos. Aquí los efectos nutracéuticos pueden considerarse indirectos, ya que un consumo regular de estos PUFA, en teoría, puede producir 
menores cantidades de grasa corporal en el tiempo y disminuir la obesidad, que puede llevar a producir hiperinsulinemia, y relacionarse con hipertensión e hiperlipidemia (Wildman, 2007).

Tabla 21

Ejemplos de grupos de nutracéuticos agrupados por mecanismo de acción

\begin{tabular}{|c|c|c|c|c|}
\hline Anticancerígenos & $\begin{array}{l}\text { Influencia positiva } \\
\text { sobre el perfil } \\
\text { lipídico en sangre }\end{array}$ & $\begin{array}{l}\text { Actividad } \\
\text { antioxidante }\end{array}$ & $\begin{array}{c}\text { Actividad } \\
\text { antiinflamatoria }\end{array}$ & $\begin{array}{l}\text { Protección } \\
\text { osteogenética }\end{array}$ \\
\hline Capsaicina & $\beta$-glucano & CLA & $\begin{array}{l}\text { Ácido linole- } \\
\text { nico }\end{array}$ & CLA \\
\hline Genisteína & g-tocotrienol & $\begin{array}{l}\text { Ácido ascór- } \\
\text { bico }\end{array}$ & EPA & $\begin{array}{l}\text { Proteína de } \\
\text { soya }\end{array}$ \\
\hline Daidzeína & d-Tocotrienol & $\beta$-caroteno & DHA & Genisteína \\
\hline$\alpha$-Tocotrienol & MUFA & Polifenoles & GLA & Daidzeína \\
\hline g-Tocotrienol & Quercetina & Tocoferoles & Capsaicina & Calcio \\
\hline CLA & $\omega-3$ PUFA & Tocotrienoles & Quercetina & $\begin{array}{l}\text { Fosfopéptidos } \\
\text { de caseína }\end{array}$ \\
\hline $\begin{array}{l}\text { Lactobacillus } \\
\text { acidophilus }\end{array}$ & Resveratrol & $\begin{array}{l}\text { Indol-3- car- } \\
\text { banol }\end{array}$ & Curcumina & FOS \\
\hline Esfingolípidos & Taninos & a- Tocoferol & & Inulina \\
\hline Limoneno & $\beta$-sitosterol & Ácido elágico & & \\
\hline Dialil disulfuro & Saponinas & Licopeno & & \\
\hline Ajoene & Guar & Luteína & & \\
\hline a- Tocoferol & Pectina & Glutatión & & \\
\hline Enterolactona & & Hidroxitirosol & & \\
\hline Glicirricina & & Luteolina & & \\
\hline Equol & & Oleuropeína & & \\
\hline Curcumina & & Catequina & & \\
\hline Ácido elágico & & Gingerol & & \\
\hline Luteína & & $\begin{array}{l}\text { Ácido cloro- } \\
\text { génico }\end{array}$ & & \\
\hline Carnosol & & Taninos & & \\
\hline L. bulgaricus & & & & \\
\hline
\end{tabular}

Fuente: (Wildman, 2007) 


\section{Clasificación según la naturaleza química}

Otra forma de clasificación de los nutracéuticos está basada en su naturaleza química. Esta clasificación permite categorizar los nutracéuticos bajo grupos moleculares/elementales. Este modelo preliminar incluye varios grupos grandes que proveen una base para subclasificación o subgrupos, y así sucesivamente. Una forma de agrupar nutracéuticos es la siguiente:

- Derivados de isoprenoides

- Sustancias fenólicas

- Ácidos grasos y lípidos estructurales

- Carbohidratos y derivados

- Sustancias basadas en aminoácidos

- Microorganismos

- Minerales

A medida que la investigación científica continúa, varios cientos de sustancias serán consideradas nutracéuticas, y se espera que los científicos reorganicen este sistema y encuentren nuevas y mejores formas de clasificación (Wildman, 2007).

\section{Compuestos Fenólicos}

Al igual que los terpenoides, los compuestos fenólicos también se consideran metabolitos secundarios. La base para esta familia tan diversa es una estructura de fenol, el cual es un anillo aromático con un grupo hidroxilo. De esta estructura, moléculas más grandes e interesantes se forman, como las antocianinas, cumarinas, fenilpropamidas flavonoides, taninos y lignina. Los compuestos fenólicos realizan una variedad de funciones para las plantas, incluyendo defensa contra los herbívoros y patógenos, atrayendo polinizadores, reduciendo el crecimiento de plantas competitivas y promo- 
viendo las relaciones simbióticas con bacterias fijadoras de nitrógeno (Bagchi, Prevs, Swaroop, 2016).

Los flavonoides son una de las clases de compuestos fenólicos más grandes en las plantas. La estructura básica de los flavonoides contiene 15 carbonos y está compuesta por 2 anillos aromáticos unidos por un puente de 3 carbonos. Los flavonoides se clasifican basados en el grado de oxidación del puente de 3 carbonos. También se pueden encontrar grupos hidroxilo en las posiciones de los carbonos 4, 5 y 7, y en otras posiciones. La unión de grupos hidroxilos y azúcares incrementan las propiedades hidrofílicas de la molécula de flavonoides, mientras que al unirse a metilésteres o unidades de isopentil modificado incrementará el carácter lipofílico.

Las antocianinas y antocianidinas son producidas por las plantas y trabajan como pigmentos colorantes. Básicamente las antocianinas son antocianidinas pero con un azúcar unido en la posición 3 del carbono 3 del puente entre los dos anillos. Estas moléculas ayudan a atraer animales para la polinización y dispersión de las semillas. Son responsables de los colores rojo, rosado, azul y violeta de muchas frutas y verduras, incluyendo arándanos, manzanas, remolacha roja, cerezas, uvas, naranjas, duraznos, ciruelas, rábanos, frambuesas y fresas. Solo 16 antocianidinas han sido identificadas en las plantas, incluyendo pelargonidina, cianidina, peonidina, delfinidina, malvidina y petunidina.

Aunque los flavonoles y las flavonas son similares a sus primos cercanos y antocianidinas, estas absorben luz, pero a longitudes de onda más cortas y así no son percibidas como color por el ojo humano. Sin embargo, pueden ser detectados por los insectos y ayudar a dirigirlos a las áreas de polinización (Wildman, 2007). 


\section{Carbohidratos y Derivados}

El derivado de la glucosa ácido ascórbico (Vitamina C) es una de las sustancias nutracéuticas más reconocidas, y es un suplemento muy popular. El ácido ascórbico funciona como un compuesto nutracéutico, principalmente como un antioxidante. Mientras que las plantas producen algunos oligosacáridos que parecen funcionar como sustancias prebióticas.

Muchas de las familias de polisacáridos de plantas no son fuente de energía disponible para los humanos, ya que son resistentes a las enzimas digestivas secretadas. Estos polisacáridos se agrupan junto con la lignina, compuesto polimérico fenólico que forma una de las familias de nutracéuticos más reconocidas, las fibras. La función de las fibras es estructural para las plantas; otro papel interesante de ciertas fibras es el de reparar tejidos después de los traumas, análogo a la cicatrización animal.

Otra familia de polisacáridos que también es valioso discutir son los glucosaminoglicanos (GAG). Estos compuestos se encuentran en los tejidos conectivos de los animales y son componentes potenciales de alimentos funcionales. Los GAG y el sulfato de condroitina son suplementos nutricionales populares, usados por personas que se están recuperando de daños en las articulaciones y que sufren de desórdenes de inflamación de las articulaciones. Los glucosaminoglicanos a menudo también se llaman mucopolisacáridos, se caracterizan por el contenido de amino-azúcares y ácido urónico. Ejemplos de glucosaminoglicanos son el ácido hialurónico y el sulfato de condroitina (Wildman, 2007).

\section{Ácidos Grasos y Lípidos Estructurales}

Muchos ácidos grasos y sus derivados han llamado la atención de 
los investigadores debido a su potencial funcional. Estos incluyen los w-3 PUFA que se encuentran en mayores concentraciones en las plantas, peces y otros animales marinos. Otros dos tipos de lípidos en los productos alimenticios son los lípidos estructurados y diglicéridos. Los lípidos estructurados son triglicéridos que han sufrido una hidrólisis y re-esterificación bajo condiciones que resultan en triglicéridos con nuevas combinaciones de ácidos grasos. (Wildman, 2007).

\section{Basados en Aminoácidos}

Este grupo tiene el potencial de incluir proteínas completas, polipéptidos, aminoácidos y derivados de aminoácidos con nitrógeno y azufre. Pocos aminoácidos han sido investigados por su potencial nutracéutico. Entre estos aminoácidos se tienen arginina, ornitina, taurina y ácido aspártico. Se especula que la arginina es un cardioprotector, y que es una molécula precursora de sustancias vasodilatadoras del óxido nítrico (NO). Otra molécula nutracéutica derivada de aminoácidos es el ácido fólico, el cual se cree que tiene un papel cardioprotector además de minimizar los niveles de homocisteína. Otros miembros de este grupo incluirían los tripéptidos glutatión y colina (Wildman, 2007).

\section{Microorganismos (Probióticos)}

Los probióticos involucran microorganismos viables. Este grupo incluye las bacterias, y su criterio es que deben ser resistentes a las condiciones ácidas del estómago, bilis y las enzimas digestivas que se encuentran normalmente en el tracto gastrointestinal humano. Entre las especies bacterianas reconocidas, que tienen potencial en un alimento funcional, están: Lactobacillus acidophilus, L. plantarum, L. casei, Bifidobacterium bifidum, B. infantis, y Streptococ- 
cus salvarius subespecie thermophilus. Algunas levaduras también han incluido, entre ellas, Saccharomyces boulardii (Keservani et al., 2010; Wildmand, 2007).

\section{Minerales}

Muchos minerales se han reconocido por su potencial nutracéutico, convirtiéndose en ingredientes candidatos para las fórmulas de alimentos funcionales. Entre los más obvios está el calcio, con relación a la salud de los huesos, cáncer de colon y, posiblemente, hipertensión y enfermedad cardiovascular. El potasio también ha sido considerado para reducir la hipertensión y así mejorar la salud cardiovascular. Unos cuantos minerales traza han sido encontrados que tienen potencial nutracéutico, estos incluyen cobre, selenio, manganeso y zinc; el potencial nutracéutico generalmente se relaciona con la capacidad antioxidante (Wildman, 2007).

\section{REVISIÓN DEL POTENCIAL DE SEIS PRODUCTOS DE LA TRADICIÓN AGRÍCOLA DEL SUR DE ATLÁNTICO COLOMBIANO COMO FUENTES DE NUTRACÉUTICOS}

\section{Ahuyama}

La ahuyama pertenece al género de cucurbita y a la familia cucurbitaceae, y cubre cultivares de cualquiera de las especies cucurbita pepo, cucurbita máxima, cucurbita moschata, provenientes de Norteamérica. Típicamente tiene una corteza gruesa naranja o amarilla, arrugas desde el vástago hasta el fondo, semillas y pulpa (Alhassan, Hashim, Ahmed, y Khogali, 2017).

En un estudio realizado en Colombia Alzate et al. (2017) presentan la ahuyama como una fuente importante de luteína, junto al repollo y la lechuga. Igualmente, se han caracterizado distintas variedades 
de ahuyama en cuanto a su contenidos de $\alpha$-caroteno, $\beta$-caroteno, luteína, zeaxantina y equivalente de retinol, hallando que las variedades más ricas en carotenos son aquellas de color anaranjado, y las más ricas en luteína las de apariencia amarilla (Murkovic, Mülleder y Neunteufl, 2002). Alhassan et al. (2017) reportan 22 compuestos bioactivos analizados por la técnica de GC-MS. Neelamma, Dura y Dhamodaran (2016) plantean una interesante revisión de estudios sobre el prospectivo uso fitoterapéutico de la ahuyama. Al-Anoos, El-dengawy y Hasanin (2015), evaluaron las semillas de cucurbita máxima encontrando ricos contenidos de proteínas y grasas, $\mathrm{Fe}$, $\mathrm{Cu}, \mathrm{Mg}$.

Zapata, Piedrahita y Rojano (2014) seleccionaron 24 frutas y 18 hortalizas colombianas para determinar el contenido de fenoles totales y capacidad ORAC. Entre los reportes para hortalizas, la ahuyama se sitúa en el grupo de bajo contenido, el pimentón y la cebolla en el de alto; para las frutas, la guayaba en el grupo de alto nivel y capacidad ORAC. El mango en cambio en el bajo.

\section{Cebolla}

Las cebollas (Allium cepa L.) son el segundo cultivo hortícola más importante del mundo (Benitez et al., 2011). La cebolla larga (Allium fistulosum L.) es una especie altamente comercializada en Colombia debido a sus atributos sensoriales. La cebolla ha demostrado ser una buena fuente de compuestos fenólicos (Parvu, Toiu, Vlase y Parvu, 2010) que potencializan su capacidad antioxidante (Piedrahita, Rojano, Zapata, 2014). Así mismo, la cebolla presenta compuestos azufrados con bioactividad, capaces de modificar positivamente los sistemas antioxidante, inflamatorio y cardiovascular (Benitez et al., 2011; Goncharov et al., 2016). 
Dos grupos principales de flavonoides encontrados en las cebollas son: antocianinas (cianidina y glicósidos de peonidina) y flavonoles (quercetina, isoramnetina, kaempferol y sus glicósidos). Los flavonoles más abundantes en las cebollas son: la quercetina 4'-O- $\beta$-D-glucósido y la quercetina 3,4'-O- $\beta$-D-diglucósido, que representan más del $85 \%$ del contenido total de flavonoides (ZiII-E-Huma et al., 2011). La quercetina presenta una alta capacidad antioxidante y ha sido empleada como complemento nutricional nutracéutica. La quercetina es 77 veces más abundante en la parte no comestible que en la parte comestible del material blanco de la cebolla (Ko, Cheigh, Cho y Chung, 2011). Algunos de los beneficios funcionales de la quercetina incluyen: actividad anti-inflamatoria, efecto antihistamínico, antialérgica, y actividades anticancerígenas y antivirales. También se ha afirmado que la quercetina reduce la presión arterial en sujetos hipertensos (Boots, Haenen y Bast, 2008; Corzo-Martínez, Corzo y Villamiel, 2007; Prakash, Singh y Upadhyay, 2007).

Los constituyentes primarios que contienen azufre en la cebolla son: sulfóxidos de S-alk(en)yl-L-cisteína, tales como Aliina, y G-glutamilcisteínas, que, además de servir como péptidos de almacenamiento, son intermedios biosintéticos para la formación de volátiles, tales como alicina y compuestos de azufre liposolubles, tales como sulfuro de dialilo, disulfuro de dialilo, entre otros (Edwards, Britton y Collin, 1994). Estos compuestos proporcionan a la cebolla su olor y sabor característicos, así como gran parte de sus propiedades biológicas (Corzo-Martínez, Corzo y Villamiel, 2007).

\begin{abstract}
Ají
El ají o pimiento es uno de los vegetales más importantes, generalmente consumido como alimento y como especia. El ají topito
\end{abstract}


(Capsicumannuum) es una variedad altamente cultivada al norte de Colombia. Muchos estudios han demostrado que los ajíes contienen una amplia gama de fitoquímicos, principalmente vitaminas $C$, A y $E$, así como compuestos fenólicos y carotenoides con propiedades antioxidantes bien conocidas (Ghasemnezhad, Sherafati y Payvast, 2011; Hallmann y Rembiałkowska, 2012; Hervert, Sayago y Goni, 2010; Ranilla, 2010). Los ajíes son una buena fuente de provitamina A ( $\beta$-caroteno, R-caroteno y $\beta$-criptoxantina) y carotenoides oxigenados o xantofilas que han demostrado ser efectivos en la eliminación de radicales libres (Hervert, 2010). El ají fresco es uno de los vegetales con más alto contenido de vitamina $C$, entre 42-93 mg/100 g. (Marín, Tomás-barberán y Gil, 2004).

Los principales compuestos fenólicos encontrados en el ají dulce (Capsicum annuum L.) han sido: quercetina-3-O-ramnosido y luteolina 7-O-(2-episodul-6-malonil) glucósido, que representan hasta el $41 \%$ de los flavonoides totales (Marín Tomás-Barberán y Gils, 2004).

Se ha encontrado que el tipo de cultivo (orgánico o tradicional), la variedad y estado de madurez pueden afectar la concentración de los compuestos bioactivos presentes en el ají, como carotenos, fenoles y vitamina C (Ghasemnezhad, Sheratati y Payvast 2011; HaIlmann y Rembialkowska, 2012; Hornero-Méndez, Gómez-Ladrón De Guevara, y Mínguez-Mosquera, 2000; Marín et al., 2004).

Los ajís sufren un cambio profundo durante el proceso de maduración debido a la transformación de los pigmentos. El color verde del fruto se debe principalmente a la presencia de clorofila y carotenoides típicos del cloroplasto, tales como carotenoides oxigenados o xantofilas, violaxantina, neoxantina y luteína, así como $\beta$-ca- 
roteno. Mientras que pigmentos carotenoides como la capsantina, capsorubina y 5, 6 -epóxido-capsantina, son responsables del color rojo final (Hornero-Méndez, Gómez y Mínguez, 2000; Marín et al., 2004).

\section{Limón}

El limón (Citrus Limón) es otra fruta producida en Colombia. El limón contiene muchos componentes químicos naturales importantes, incluidos compuestos fenólicos, principalmente flavonoides, y nutrientes de alto valor como vitaminas (B9 y C), minerales (potasio), fibra dietaria y carotenoides, así como aceites esenciales (Gonzales, Dominguez, Moreno y Garcia, 2010; Jideani y Jideani, 2012). Sus efectos y propiedades promotoras de la salud se han asociado con sus contenidos de vitamina $\mathrm{C}$ y flavonoides, debido a sus características antioxidantes naturales (Gattuso et al., 2007; Gonzales et al., 2010; Wang, Chuang y Ku, 2007). En general, las frutas de limón, ricas en flavonoides, son una parte muy importante de una dieta equilibrada, en particular por su papel en la prevención de enfermedades, como la obesidad, la diabetes, la reducción de lípidos en la sangre, las enfermedades cardiovasculares y ciertos tipos de cáncer (Gonzales, Domínguez, Moreno y García, 2010; Jideani y Jideani, 2012).

Estudios han referido la producción de cítricos, como de las que más subproductos genera, cerca del $50 \%$, de la industria de jugos, mermeladas y conservas. La caracterización de esos subproductos los presentan como sustratos de bajo costo para la obtención de compuestos fenólicos y de fibra dietaría; esta última con muchos reportes de aplicación en matrices alimentarias (Alarcón, López y Restrepo, 2015). 


\section{Mango}

El mango (Mangifera indica L.) es una de las frutas tropicales más apreciadas e importantes que se consume en forma fresca o procesada a nivel mundial (Gill, 2016). Existen más de mil diferentes cultivos de mangos creciendo en todo el mundo. El mango es conocido por ser una excelente fuente de nutrientes y vitaminas, como el ácido ascórbico, tiamina, riboflavina, niacina y ß-caroteno. La cáscara de mango ha demostrado ser una buena fuente de compuestos fenólicos, carotenoides y vitamina E (Ajila, Naidu, Bhat y Rao, 2007; Ajila, Rao y Rao, 2010); igualmente es una buena fuente de fibra dietaria (Ajila, Aalami, Leelavathi y Rao, 2010).

El mango azucarero (Mangifera indica), cultivado en Colombia, ha demostrado ser una buena fuente de carotenoides y flavonoides, presentando además una alta capacidad antioxidante (Corrales, Maldonado, Urrango, Franco, y Rojano, 2014).

Los ácidos clorogénico, gálico, vanílico y protocatecuico han sido referenciados como los principales compuestos fenólicos en la pulpa de mango, y tienden a aumentar con la maduración del fruto, siendo el ácido clorogénico el más abundante. Así mismo, se ha encontrado que la contribución a la capacidad antioxidante de estos cuatro ácidos aumenta durante la maduración. El ácido gálico presenta la mayor contribución antioxidante, seguido por el ácido clorogénico (Palafox-Carlos, Yahia y González-Aguilar, 2012).

En cuanto a la industrialización del mango, los subproductos están comprendidos por la piel (10-20\%), semilla (10-25\%). Autores presentan el potencial de la semilla para la extracción de aceite de alto nivel de insaturación (Alarcón García, López Vargas y Restrepo Molina, 2015), y altos niveles de polifenoles y carotenoides en 
la piel del mango en dependencia de su grado de madurez. Los niveles de fibra dietaria en la cáscara del mango fluctúan entre el $44 \%$ y $78 \%$. Estudios muestran el uso potencial de estas fibras en alimentos comunes como las galletas (Alarcón García, López y Restrepo, 2015).

\section{Guayaba}

La guayaba (Psidium guajava L.) es considerada como uno de los cultivos frutales más exquisitos, nutricionalmente valiosos y remunerativos de las regiones tropicales y subtropicales del mundo (Gill, 2016). Botánicamente, el fruto de la guayaba es una baya; su forma puede variar de redonda a oblonga, ovalada o pera. Puede tener cáscara áspera o lisa y con semillas pequeñas, duras o semiduras incrustadas al interior de la pulpa. El color de la cáscara, en frutos maduros, varía de verde pálido a color amarillo brillante, con o sin tonalidad rojiza (Gill, 2016; Restrepo-Sanchez, Narvaez-Cuenca y Restrepo-Sanchez, 2009).

La guayaba está compuesta de $20 \%$ de cáscara, $50 \%$ pulpa y $30 \%$ de semilla, tiene un bajo valor calórico (68 kcal./100 g.) y un contenido bajo de grasa y proteína (Gill, 2016). Nutricionalmente, la guayaba es una fruta de alto valor, ya que es buena fuente de calcio (18 mg./100 g. fruta fresca) y bastante rica en fósforo (40 mg./100 g. fruta fresca) (Gill, 2016; López, Dupas, Kawazoe y Santos, 2012). Sus frutos contienen altas cantidades de vitaminas A, B1 (tiamina), B2 (riboflavina) y C (ácido ascórbico). El contenido de vitamina C de la guayaba es 2-5 veces más que el de los cítricos. El contenido típico de Vitamina C oscila entre $50-400 \mathrm{mg} / 100 \mathrm{~g}$. de fruta fresca (López et al., 2012; Ramírez y Pacheco de Delahaye, 2011; Restrepo-Sanchez et al., 2009). Recientemente, un estudio realiza- 
do por Contreras-Calderón, Calderón-Jaimes, Guerra-Hernández y García-Villanova (2011) reportó un contenido de 257 mg./100 g. en Guayaba manzana (Híbrido de psidium guajava) y $102 \mathrm{mg} . / 100 \mathrm{~g}$. Guayaba agria (psidium araca) cultivadas en Colombia.

De igual manera, la guayaba es una alta fuente de fibra dietaria, con un contenido del 5 \% en fruta fresca (Gill, 2016; Jiminez-Escrig, Rincon, Pulido, y Saura-Calixto, 2001) Posee una alta concentración de pectina. La guayaba puede desempeñar un papel importante en la reducción del colesterol y, por lo tanto, disminuye el riesgo de enfermedad cardiovascular (Gill, 2016).

La guayaba es una excelente fuente de antioxidantes dietéticos, tales como los polifenoles y los carotenoides. Los compuestos de polifenoles se encuentran principalmente en la cáscara y pulpa, contribuyendo significativamente a la alta capacidad antioxidante de la guayaba. La guayaba roja es una buena fuente de carotenoides antioxidantes (criptoxantina, licopeno y $\beta$-caroteno), y contiene aproximadamente $140 \mu \mathrm{g}$ de equivalentes de retinol/100 g. de provitamina $\mathrm{A}$; otros autores han categorizado a la guayaba como una fuente rica de licopeno, con alrededor del $80 \%$ de los compuestos carotenoides (Gill, 2016; López et al., 2012).

El contenido de fenoles totales en diferentes variedades de guayaba oscila entre 190 a $450 \mathrm{mg}$. de ácido gálico/100 g. La actividad antioxidante medida por FRAP está entre 80 a $150 \mu \mathrm{mol}$ Trolox/g. y por ABTS se ha reportado un valor de 40 a $80 \mu \mathrm{mol}$ Trolox/g. fruta fresca (Restrepo-Sanchez et al., 2009). Adicionalmente, se han encontrado valores FRAP de 40 y $62 \mu \mathrm{mol}$ Trolox/g. para guayaba agria y manzana respectivamente, y valores ABTS de 45 y $50 \mu \mathrm{mol}$ Trolox/g. para Guayaba agria y manzana respectivamente, cultivadas en Colombia (Contreras-Calderón et al., 2011). 
Recientemente se encontró un contenido de fenoles de $385 \mathrm{mg} . /$ $100 \mathrm{~g}$. fruta fresca, $502 \mathrm{mg}$. de tanino/100 g. fruta fresca y 133 mg. de Flavonoides/100 g. fruta fresca para guayaba agria (Psidium araca) cultivada en Colombia (Zapata, Cortes y Rojano, 2013). Asimismo se halló un valor ABTS de $6679 \mu \mathrm{mol}$ Trolox/g. fruta fresca y FRAP de $623 \mathrm{mg}$. Ácido Ascórbico equivalente/100 g. de fruta fresca, demostrando un alto potencial antioxidante de esta variedad de guayaba cultivada en Colombia (Zapata et al., 2013).

Por otra parte, las semillas de guayaba han sido de particular interés en los últimos años, ya que son un subproducto de la industria y tienen un alto valor nutricional. Se ha encontrado en semillas deshidratadas contenidos de $63 \mathrm{~g}$. de fibra dietaria, $11 \mathrm{~g}$. de proteína y 13 de lípidos en $100 \mathrm{~g}$ de semilla deshidratada. De la fracción lipídica de las semillas, un 77 \% está constituida por ácido linoleico. Asimismo, se ha encontrado un contenido de vitamina $C$ de 87 mg./100 g. (Mohammed, 2015; Uchôa-thomaz et al., 2014).

\section{CONCLUSIONES}

A medida que la investigación científica continúe, varios cientos de sustancias serán consideradas nutracéuticas y se espera que surjan nuevas propuestas de clasificación, así como la evidencia científica de sus mecanismos de acción y roles en el mejoramiento de la salud humana.

Son cada vez más los ejemplos de escalamientos de la investigación básica a la aplicada en el desarrollo de extractos completos y de alimentos funcionales, que buscan probar a nivel in vivo su efectividad. 
El potencial de las fuentes completas, así como el aprovechamiento de los subproductos de procesos de industrialización (cáscaras y semillas) para la obtención de ingredientes nutracéuticos, no solo serían una estrategia viable para mitigar los impactos ambientales, sino que sumarían potencialidad y valor agregado para las asociaciones y cooperativas que agrupan a los cultivadores del sur del Atlántico.

\section{REFERENCIAS BIBLIOGRÁFICAS}

Ajila, C. M., Aalami, M., Leelavathi, K. y Rao, U. J. S. P. (2010). Mango peel powder: A potential source of antioxidant and dietary $\mathrm{fi}$ ber in macaroni preparations. Innovative Food Science and Emerging Technologies, 11, 219-224. http://doi.org/10.1016/j. ifset.2009.10.004

Ajila, C. M., Naidu, K. A., Bhat, S. G. y Rao, U. J. S. P. (2007). Food Chemistry Bioactive compounds and antioxidant potential of mango peel extract, 105, 982-988. http://doi.org/10.1016/j. foodchem.2007.04.052

Ajila, C. M., Rao, L. J. y Rao, U. J. S. P. (2010). Characterization of bioactive compounds from raw and ripe Mangifera indica L. peel extracts. Food and Chemical Toxicology, 48(12), 3406-3411. http://doi.org/10.1016/j.fct.2010.09.012

Al-Anoos, I., El-dengawy, R. y Hasanin, H. (2015). Studies on Chemical Composition of Some Egyptian and Chinese pumpkin (Cucurbita maxima) Seed Varieties. Journal of Plant Science \& Research, 2(2), 1-4.

Alarcón García, M., López Vargas, J. H. y Restrepo Molina, D. A. (2015). Agro-industrial fruit co-products in Colombia, their sources and potential uses in processed food industries: a review. Revista Facultad Nacional de Agronomía, 68(2), 77297742. http://doi.org/10.15446/rfnam.v68n2.50993 
Alhassan, A. M., Hashim, H., Ahmed, S. y Khogali, M. E. (2017). GC-ms Analysis Report About Pumpkin Fruit (Cucurbita maxima, Cucurbitaceae). International Journal of Agriculture and Environmental Research, 3(1), 2025-2039.

Alzate T. L. M., Gonzalez, D., Hincapi, S., Cardona S, B. L., Londoño-Londoño, J. y Jiminez-Cartagena, C. (2017). The profile of bioactive substances in ten vegetable and fruit by-products from a food supply chain in Colombia. Sustainable Production and Consumption, 9, 37-43. http://doi.org/10.1016/j. spc.2016.07.005

Bagchi, D., Preus, H. G. y Swaroop, A. (2016). Nutraceuticals and Functional Foods in Human Health and Disease Prevention. (D. Bagchi, H. G. Preus, y A. Swaroop, Eds.) (1st ed.). Boca Raton: CRC Press. http://doi.org/10.1201/b19308

Benitez, V., Molla, E., Martin, A., Lopez, F., Downes, K., Terry, L. y Esteban, R. (2011). Study of bioactive compound content in different onion sections. Plant Foods for Human Nutrition, 66(1), 48-57.

Boots, A. W., Haenen, G. R. M. M. y Bast, A. (2008). Health effects of quercetin: From antioxidant to nutraceutical. European Journal of Pharmacology, 585(2-3), 325-337. https://doi.org/10.1016/j. ejphar.2008.03.008

Contreras-Calderón, J., Calderón-Jaimes, L., Guerra-Hernández, E. y García-Villanova, B. (2011). Antioxidant capacity, phenolic content and vitamin $C$ in pulp, peel and seed from 24 exotic fruits from Colombia. Food Research International, 44(7), 20472053. http://doi.org/10.1016/j.foodres.2010.11.003

Corrales, A., Maldonado, M., Urrango, L., Franco, M., y Rojano, B. (2014). Mango de azúcar (Mangifera indica), variedad de Colombia: características antioxidantes, nutricionales y sensoriales 
Sugar mango (Mangifera indica), variety from Colombia: antioxidant, nutritional and sensorial characteristics. Revista Chilena de Nutrición, 41(21).

Corzo-Martínez, M., Corzo, N. y Villamiel, M. (2007). Biological properties of onions and garlic. Trends in Food Science and Technology, 18(12), 609-625. https://doi.org/10.1016/j.tifs.2007.07.011

Edwards, S. J., Britton, G. y Collin, H. A. (1994). The biosynthetic pathway of the S-alk (en) yl-L-cysteine (flavour precursors) in species of AUium sulphoxides. Plant Cell, Tissue and Organ Culture, 38, 181-188. https://doi.org/10.1007/BF00033876

Farmer, F. (2005). Product development for the food ingredient industry. In G. Fuller (Ed.), New Food Product Development (Second Edi, 285-316). Boca Raton: CRC Press.

Gattuso, G., Barreca, D., Gargiulli, C., Leuzzi, U., Caristi, C., Organica, C., Sperone, S. (2007). Flavonoid Composition of Citrus Juices. Molecules, 12, 1641-1673.

Ghasemnezhad, M., Sherafati, M. y Payvast, G. A. (2011). Variation in phenolic compounds, ascorbic acid and antioxidant activity of five coloured bell pepper (Capsicum annum) fruits at two different harvest times. Journal of Functional Foods, 3(1), 44-49. http://doi.org/10.1016/j.jff.2011.02.002

Gill, K. (2016). Guavas. In B. Caballero, P. Finglas, y F. Toldra (Eds.). Encyclopedia of Food and Health,106-114. Oxford: Academic Press. http://doi.org/10.1016/B978-0-12-384947-2.00020-9

Godfray, H. C. J., Crute, I. R., Haddad , L., Lawrence, D., Muir, J.F., Nisbett, N., Pretty, J., Robinson S., Toulmin, C. and Whiteley, R. (2010). The future of the global food system. Philosophical Transactions of the Royal Society B: Biological Sciences, 365(1554), 2769-2777. http://doi.org/10.1098/rstb.2010.0180

Goncharov, N., Orekhov, A. N., Voitenko, N., Ukolov, A., Jenkins, 
R. y Avdonin, P. (2016). Organosulfur Compounds as Nutraceuticals. Nutraceuticals. Elsevier Inc. http://doi.org/10.1016/ B978-0-12-802147-7.00041-3

Gonzales, E., Dominguez, R., Moreno, D. y Garcia, C. (2010). Natural bioactive compounds of Citrus lemon for food and health. Journal of Pharmaceutical and Biomedical Analysis, 51, 327-345. http://doi.org/10.1016/j.jpba.2009.07.027

Hallmann, E. y Rembiałkowska, E. (2012). Characterisation of antioxidant compounds in sweet bell pepper (Capsicum annuum L.) under organic and conventional growing systems. Journal of the Science of Food and Agriculture, (September). http://doi. org/10.1002/jsfa.5624

Hervert, D., Sayago, S. y Goni, I. (2010). Bioactive Compounds of Four Hot Pepper Varieties (Capsicum annuum L.), Antioxidant Capacity, and Intestinal Bioaccessibility. Journal of Agricultural and Food Chemistry, 58, 3399-3406. http://doi.org/10.1021/ jf904220w

Hornero-Méndez, D., Gómez-Ladrón De Guevara, R. y Mínguez-Mosquera, M. I. (2000). Carotenoid biosynthesis changes in five red pepper (Capsicum annuum L.) cultivars during ripening. Cultivar selection for breeding. Journal of Agricultural and Food Chemistry, 48, 3857-64. https://doi.org/10.1021/jf991020r Jideani, V. A. y Jideani, I. A. (2012). Lemon and Lime. In M. Siddiq (Ed.). Tropical and Subtropical Fruits Postharvest Physiology, Processing and Packaging, 377-398. New Delhi: Jhon Wiley y Sons, Inc. http://doi.org/10.1016/j.ifset.2007.04.001 Jiminez-Escrig, A., Rincon, M., Pulido, R. y Saura-Calixto, F. (2001). Guava fruit (Psidium guajava L.) as a new source of antioxidant dietary fiber. Journal of Agricultural and Food Chemistry, 49(11), 5489-5493. http://doi.org/10.1021/jf010147p

Kaur, S. y Das, M. (2011). Functional foods: An overview. Food 
Science and Biotechnology, 20(4), 861-875. http://doi. org/10.1007/s10068-011-0121-7

Keservani, R. K., Kesharwani, R. K., Vyas, N., Jain, S., Raghuvanshi, R. y Sharma, A. K. (2010). Nutraceutical and Functional Food As Future Food: A Review. Der Pharmacia Lettre, 2(1), 106-116.

Ko, M. J., Cheigh, C. I., Cho, S. W. y Chung, M. S. (2011). Subcritical water extraction of flavonol quercetin from onion skin. Journal of Food Engineering, 102(4), 327-333. https://doi.org/10.1016/j. jfoodeng.2010.09.008

López, R., Dupas, M., Kawazoe, A. y Santos, G. (2012). Guava. In M. Siddiq (Ed.), Tropical and Subtropical Fruits Postharvest Physiology, Processing and Packaging, 469-477. New Delhi: Jhon Wiley y Sons, Inc. http://doi.org/10.1016/j.ifset.2007.04.001

Malik, V. S., Willett, W. C. y Hu, F. B. (2013). Global obesity: trends, risk factors and policy implications. Nature Reviews. Endocrinology, 9(1), 13-27. http://doi.org/10.1038/nrendo.2012.199

Marín, A., Tomás-barberán, F. A. y Gil, M. (2004). Characterization and quantitation of antioxidant constituents of sweet pepper (Capsicum annuum L.). Journal of Agricultural and Chemistry, 52, 3861-3869. https://doi.org/10.1021/jf0497915

Mohammed, A. (2015). Nutritional composition, antinutritional factors, bioactive compounds and antioxidant activity of guava seeds (Psidium Myrtaceae) as affected by roasting processes. Journal of Food Science Tecnology, 52(April), 2175-2183. http:// doi.org/10.1007/s13197-013-1242-1

Murkovic, M., Mülleder, U. y Neunteufl, H. (2002). Carotenoid Content in Different Varieties of Pumpkins. Journal of Food Composition and Analysis, 15(6), 633-638. http://doi.org/10.1006/ jfca.2002.1052

Neelamma, G., Dura, B. y Dhamodaran, P. (2016). Phytochemical 
and pharmacological overview of cucurbita maxima and future perspective as potential phytotherapeutic agent. European Journal of Pharmaceutical and Medical Research, 3(8), 277-287.

Palafox-Carlos, H., Yahia, E. M. y González-Aguilar, G. A. (2012). Identification and quantification of major phenolic compounds from mango (Mangifera indica, cv. Ataulfo) fruit by HPLC-DADMS/MS-ESI and their individual contribution to the antioxidant activity during ripening. Food Chemistry, 135(1), 105-111. https://doi.org/10.1016/j.foodchem.2012.04.103

Parvu, M., Toiu, A., Vlase, L. y Parvu, E. A. (2010). Natural Product Research: Formerly Natural Product Letters Determination of some polyphenolic compounds from Allium species by. Natural Product Research: Formerly Natural Product Letters, (October, 2013), 37-41. http://doi.org/10.1080/14786410903309484

Pérez-Álvarez, J. A., Sayas-Barberá, E. \& Fernandez-López, J. (2003). La alimentación en las sociedades occidentales. En: J. Peréz-Álvarez, E. Sayas-Barberá, J. Fernández-López. Alimentos funcionales y dieta Mediterránea. Elche: Univ. Miguel Hernández. pp.11-25.

Prabu, S. L., Suriyaprakash, T. N. K., Kumar, C. D., Sureshkumar, S. y Ragavendran, T. (2012). Nutraceuticals: A review. Elixir Pharmacy, 46, 8372-8377.

Prakash, D., Singh, B. N. y Upadhyay, G. (2007). Antioxidant and free radical scavenging activities of phenols from onion (Allium cepa). Food Chemistry, 102(4), 1389-1393. https://doi.org/10.1016/j.foodchem.2006.06.063

Ramírez, A. y Pacheco de Delahaye, E. (2011). Composición química y compuestos bioactivos presentes en pulpas de piña, guayaba y guanábana. Interciencia, 36(1), 71-75.

Ranilla, L. G. (2010). Phenolic compounds, antioxidant activity 
and in vitro inhibitory potential against key enzymes relevant for hyperglycemia and hypertension of commonly used medicinal plants, herbs and spices in Latin America. Bioresource Technology, 101(12), 4676-4689. http://doi.org/10.1016/j. biortech.2010.01.093

Restrepo-Sánchez, D. C., Narvaez-Cuenca, C. E. y Restrepo-Sánchez, L. P. (2009). Extracción de compuestos con actividad antioxidante de frutos de guayaba cultivada en Vélez-Santander, Colombia. Quimica Nova, 32(6), 1517-1522. http://doi. org/10.1590/S0100-40422009000600030

Shahidi, F. (2009). Nutraceuticals and functional foods: Whole versus processed foods. Trends in Food Science and Technology, 20(9), 376-387. http://doi.org/10.1016/j.tifs.2008.08.004

Uchôa-thomaz, A. M. A., Sousa, E. C., Carioca, J. O. B., Morais, S. M. De, Lima, A. De, Martins, C. G., Rodrigues, L. L. (2014). Chemical composition, fatty acid profile and bioactive compounds of guava seeds (Psidium guajava L.). Food Science and Technology (Campinas), 34(3), 485-492. http://doi.org/10.1590/1678-457x.6339

Wang, Y., Chuang, Y. y Ku, Y. (2007). Quantitation of bioactive compounds in citrus fruits cultivated in Taiwan. Food Chemistry, 102, 1163-1171. http://doi.org/10.1016/j.foodchem.2006.06.057

Wildman, R. E. C. (2001). Handbook of nutraceuticals and functional foods. R. E. C. Wildman (Ed.). Boca Raton, Fla.: CRC Press.

Wildman, R. E. C. (2007). Handbook of nutraceuticals and functional foods. R. E. C. Wildman (2d. Ed.). Boca Raton, Fla.: CRC Press.

Zapata, K., Cortés, F. B. y Rojano, B. A. (2013). Polifenoles y Actividad Antioxidante del Fruto de Guayaba Agria (Psidium araca). Información Tecnológica, 24(5), 103-112. http://doi.org/10.4067/ S0718-07642013000500012

Zapata, S., Piedrahita, A. y Rojano, B. (2014). Capacidad atrapa- 
dora de radicales oxígeno (ORAC) y fenoles totales de frutas y hortalizas de Colombia. Perspectivas en Nutrición Humana, 16, 25-36.

Zill-E-Huma, Vian, M. A., Fabiano-Tixier, A. S., Elmaataoui, M., Dangles, O. y Chemat, F. (2011). A remarkable influence of microwave extraction: Enhancement of antioxidant activity of extracted onion varieties. Food Chemistry, 127(4), 1472-1480. https://doi.org/10.1016/j.foodchem.2011.01.112

\section{Cómo citar en este capítulo}

Restrepo Flórez, C. E., Estrada-López, H. H., \& Saumett-España, H. G. (2017). Nutracéuticos y alimentos funcionales: una revisión de oportunidades. En H. H. Estrada-López, H. G. Saumett-España, M. A. Iglesias-Navas, M. J. Bahamón, A. M. Cáceres-Martelo, C. E. Restrepo Flórez, . . . A. Díaz Pérez, Productos de confitería nutracéutica. Una opción empresarial para cultivadores de frutas y hortalizas (pp.141-178). Barranquilla: Universidad Simón Bolivar. 